\title{
INVESTIGACIÓN INTERDISCIPLINARIA EN LAS ARTES Y EL DISEÑO
}

\section{INTERDISCIPLINARY RESEARCH IN ARTS AND DESIGN.}

\section{MARÍA ISABEL DE JESÚS TÉLLEZ GARCÍA}

Doctor en Imagen, Tecnología y Diseño, magíster en Escenografía por la Universidad Complutense de Madrid, licenciada en Diseño Industrial por la Universidad de La Salle Bajío campus León y licenciada en Música Cantante por la Universidad de Guanajuato. Responsable del Cuerpo Académico Diseño y Artes con LGAC Teoría y práctica del diseño y las artes. Adscrita al Departamento de Diseño de la División de Arquitectura, Arte y Diseño del Campus Guanajuato de la Universidad de Guanajuato (México). Dirección: Luis Ponce de León No. 32. Mineral de la Hacienda. CP. 36251. Guanajuato, (Guanajuato).

isabeltellez@gmail.com

\section{RESUMEN:}

La investigación académica en la actualidad no solo exige el empleo de la metodología científica con resultados que realicen aportes al conocimiento, de la misma forma reclama el trabajo interdisciplinario con objeto de enriquecer las derivaciones obtenidas sobre un tema señalado. Este tipo de trabajo es posible encontrarlo desde tiempo atrás en el área de las ciencias exactas y las ciencias naturales, profesiones con tradición en el desarrollo de la investigación científica. No es el caso del área de las artes, que actualmente busca incorporar e impulsar esta actividad: En este sentido, el problema de su asimilación, las exigencias que su desarrollo conlleva y sus necesidades son aspectos presentes a lo largo del desarrollo de esta reflexión.

\section{PALABRAS CLAVE:}

Interdisciplina, investigación, cuerpos académicos, diseño.

\section{ABSTRACT:}

Academic research requires not only the use of scientific methodology with results that made contributions to knowledge, in the same way today calls for interdisciplinary work with the aim of enriching the leads obtained on a designated topic. This type of work it is possible to find it from long ago in the area of exact sciences and natural sciences, professions with tradition in the development of scientific research. It is not the case of the area of the arts which currently seeks to incorporate and promote this activity. In this sense, the problem of assimilation, demands that its development involves and their needs are present throughout the development of this reflection.

\section{KEYWORDS:}

Interdisciplinarity, Academic Bodies, Research, Design. 


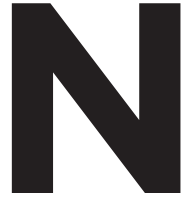
o es posible iniciar una reflexión en torno a la investigación científica y el trabajo interdisciplinario si no se pone en claro el concepto de interdisciplina. Se entiende por interdisciplina aquella actividad que para su desarrollo involucra investigadores, profesores y alumnos de diversas especialidades, integrados en un equipo que busca un fin común, y que al final del proceso logra una visión plural de un hecho o problema, de la cual se derivan resultados más envolventes, evitando la obtención de datos dispersos o fragmentados. Teniendo como base este concepto, este estudio reflexiona sobre la necesidad de incluir la investigación interdisciplinaria en el área de las artes, específicamente del diseño, y de las necesidades que su práctica requiere, así como las implicaciones que tiene omitir esta actividad en el trabajo académico actual.

Aclarado el concepto que si va a estudiar, es importante analizar el proceso de formación y trabajo del profesional o académico perteneciente al ramo de las artes; en las siguientes disciplinas: música, artes visuales, arquitectura, o diseño, entre otras, generalmente la enseñanza del arte implica una concepción diferente que plantea la adquisión del conocimiento de manera individual en dependencia del talento del estudiante. El profesional se presenta en escena, en la obra, o en el museo solo, respecto a esta observación es posible afirmar que se trata de un especialista acostumbrado a desempeñarse en solitario, sin embargo, cuenta con tareas en las que debe lidiar con otras profesiones para el logro de sus objetivo; si lo analizamos con calma, es fácil detectar que un cantante requiere de una orquesta o un pianista que lo acompañe, que un arquitecto cuenta con un equipo de trabajo el cual coordina para llevar a efecto aquello que trazó solo en su computadora, que el diseñador necesita de técnicos y profesiones que facilitarán la entrega de sus productos ya sea visuales o físicos; estos son solo algunos ejemplos, además, es posible encontrar que la mayoría de los artistas requiere de otros elementos para realizar su trabajo.

Hasta este momento hemos expuesto la realización de actividades prácticas; si pasamos al plano intelectual, teniendo como escenario la universidad en la cual conviven, se forman y desarrollan varias profesiones, es necesario plantear seguidamente interrogantes entorno al objeto de estudio: ¿Será posible encontrar correspondencia entre diversas disciplinas en el trabajo académico o resulta de mayor complejidad la integración y desarrollo común de investigadores de varias especialidades? ¿Cómo se da el diálogo entre disciplinas en un proceso de investigación? ¿Qué condiciones exige de los investigadores la investigación interdisciplinaria? para facilitar la reflexión y análisis delimitaremos su desarrollo al área de desempeño del diseñador en el ámbito académico.

Los estudios que probablemente inician el debate son los presentados en el Boletín Informativo del Programa de Inves- tigación Estratégica de Bolivia en junio de 2008 a través de una serie de pequeños artículos dedicados a la necesidad de la investigación interdisciplinaria en el área de las ciencias socia-les. Aunque los mismos hacen referencia a un área de estudio diferente, constituyen el punto de partida en la realización de planteamientos correspondientes al área de las artes. Otra investigación de importancia es el artículo "Evolución de los cuerpos académicos en la Universidad de Guanajuato"; en ella se destaca el valor de los grupos de investigación integrados por diversas disciplinas y su jerarquía en el logro de las metas de la institución. Una vez analizada la bibliografía disponible se ha corroborado que existe un área de oportunidad en el estudio de la investigación interdisciplinaria en las artes y el diseño, cuyo desarrollo se presenta a continuación.

\section{DESARROLLO}

A lo largo de su historia el diseño se ha ocupado de los objetos y sus relaciones con los procesos de cambio. La creciente importancia del diseño y su vinculación con los últimos avances en estudios culturales, sociales, económicos, políticos y geográficos, aunados a la vertiginosa evolución tecnológica, propician que la práctica del diseño requiera de grupos interdisciplinares de investigación. En la actualidad, las universidades buscan incorporar este tipo de trabajo a través de la formación de grupos de investigación; grupos que en México se conocen con el nombre de "cuerpos académicos"1, los cuales constituyen un área de gran importancia en la solidez de las instituciones de educación superior, debido a las posibilidades que ofrecen a sus profesores para realizar investigación de calidad y contribuir, de esta manera, al cumplimiento de metas institucionales a través del desarrollo de sus líneas de generación y aplicación del conocimiento, sin embargo, es necesario tomar en cuenta un aspecto esencial en el cumplimiento de estas metas, el cual radica en la posibilidad que estos grupos tienen para realizar investigación desde diferentes disciplinas sobre un mismo objeto de estudio, es decir, enriquecer el conocimiento científico basado en un proceso de encuentros, diálogos e interacciones entre profesionales de diversas áreas. Es en este punto que resulta conveniente analizar las características de la investigación interdisciplinaria que permitan definir si efectivamente el diseño es una disciplina afín a esta práctica.

Recordemos que "interdisciplinario" es todo aquello cuya concepción requiere cruzar las fronteras tradicionales entre varias disciplinas académicas o entre varias escuelas del pensamiento con objeto de atender y solucionar nuevas necesidades. En aras de contribuir a formación de una idea acerca de las implicaciones de la omisión de investigar de manera

Para mayor información al respecto, confróntese: Cuerpos Académicos. Dirección de Apoyo Investigación y al Posgrado. Universidad de Guanajuato DAIP-DCAS. Disponible en: http:// ww.ugto.mx/investigacionyposgrado/promep/ca 
interdisciplinaria, el Boletín Informativo Nexos describe las posibles consecuencias que trae realizar investigaciones que contemplan un solo punto de vista en la solución de un problema social:

"... en diferentes momentos del pasado siglo, tanto la formación superior como la investigación estuvieron agrupadas en disciplinas aisladas y dispersas, situación que condujo a miradas fraccionadas, verticales y a veces reduccionistas de los hechos sociales" (Sandoval, 2008, p. 2).

Sin embargo, las condiciones de la sociedad en la actualidad han cambiado de forma vertiginosa; el siglo XXI se caracteriza por continuos avances tecnológicos, económicos, culturales y sociales, producto de la globalización. Estos efectos, aunados a la creciente comunicación entre los distintos países del mundo, ha traído consigo nuevas exigencias que traducidas al ámbito académico demandan que los investigadores superen las lecturas y análisis monodisciplinarios de la realidad y produzcan conocimientos científicos integrales.

Por su parte, Godofredo Sandoval (Director del programa de Investigación Estratégica en Bolivia [PIEB]), enfatiza que el punto central de mayor interés en la investigación interdisciplinaria radica en los planteamientos realizados desde diferentes disciplinas sobre un mismo sujeto de estudio, preguntas que hacen referencia a diversos enfoques teóricos y metodológicos para alcanzar conocimientos integrales de los problemas. Además destaca que la interdisciplinariedad en la investigación no es solo un modo de encarar el análisis de los fenómenos, sino también una actitud que en la práctica se caracteriza por la disposición de los investigadores para hacer realidad la integración de saberes científicos.

Hasta este punto queda clara la necesidad y ventajas que es posible obtener a partir del desarrollo de la investigación interdisciplinaria; por otra parte, existen dificultades para su implementación; en este aspecto Fernando Mayorga (2008), sociólogo, cientista político e investigador subraya dos obstáculos en el avance del diálogo entre disciplinas de la siguiente manera:

"Si bien hay esfuerzos de integrar equipos con profesionales de distintas disciplinas, los procesos investigativos no asumen necesariamente ese carácter, y esto se debe en alguna medida a que las disciplinas -por separado- tampoco tienen un desarrollo muy consistente en cuanto al debate teórico metodológico. Un segundo obstáculo estaría en la cultura intelectual o hábitos de trabajo... escasa predisposición o hábito que tenemos para el trabajo en equipo. Éste implica crítica, autocrítica, la apertura, revisión de posiciones..." (p. 4).
En este sentido no menos importantes son las instituciones, sus políticas e infraestructura para el desarrollo interdisciplinario; como la eficacia de sus resultados depende de forma recíproca de la calidad de sus disciplinas, es indispensable que las futuras generaciones de investigadores posean una buena formación y experiencia en la elaboración de investigaciones cuyos problemas faciliten avanzar en el cuestionamiento de las disciplinas y su consolidación (véase Lizárraga, 2008, p. 5).

En este sentido Juan Acha (2007) infiere que el diseño en cualquiera de sus especialidades, es producto de dos desarrollos entrecruzados y singulares: el tecnológico y el artístico; estos, a su vez, son resultado de la confluencia de cuatro procesos: la tendencia expansiva y vital del capitalismo, los avances de la tecnología comunicativa, con la fotografía como cúspide y como matriz de los audiovisuales, incluidos el cine y la televisión, y por supuesto, de algunos diseños, los esfuerzos del estado burgués por arrebatarle a la iglesia el poder ideológico y dotarse de recursos eficaces para persuadir y manipular a las masas, y finalmente los esfuerzos de la industria por embellecer sus productos (Para ampliar información consúltese Acha, 2006, p. 14).

A partir de la reflexión de Acha es posible establecer con cierta certeza el gran número de disciplinas que pueden intervenir en el proceso de creación de un nuevo producto, desde los inicios de bocetaje hasta que llega al mercado; así se puede derivar que el trabajo interdisciplinario en diseño es real y cada vez más necesario debido a los cambios acelerados que sufrimos constantemente. Sin embargo, Acha sostiene esta afirmación de la siguiente manera:

"... será necesario hacer hincapié en la dependencia de la evolución de cada uno de los diseños en relación con las artes y las artesanías, las ciencias y las tecnologías, asi como también con las influencias de la sociedad, del sistema estético y del individuo en la producción de diseños y en el consumo de los productos diseñados." (p. 16)

Esta dependencia requiere para la solución de nuevos problemas de la participación de varias disciplinas y la aplicación de su conocimiento en el desarrollo de un mismo objetivo. Probablemente es a partir de la formación de cuerpos académicos ${ }^{2}$ y sus redes ${ }^{3}$ que la investigación interdisciplinaria comienza a tomar un rumbo y fortalecimiento adecuado. Es importante destacar que estos grupos establecen además un instrumento de profesionalización y constante actualización del profesorado gracias a la investigación que realizan, lo cual favorece

2

Para mayor información acerca de la evolución de los Cuerpos Académicos en la Universidad de Guanajuato consúltese: Martínez, Rico Venegas y PreciadoTarabay (2006, pp. 15-24),

Conjuntos de profesores-investigadores que comparten una o más líneas afines de investigación, cuyos objetivos y metas están destinados a la generación y /o aplicación de nuevos conocimientos. 
las condiciones para enfrentar las crecientes exigencias en la formación de recursos humanos.

En el área de Artes de la Universidad de Guanajuato se realizan constantemente intentos por desarrollar actividades interdisciplinares basadas en la aplicación de una metodología científica e impulsadas por el objetivo de enriquecer el trabajo a partir de un enfoque que involucre otras miradas y perspectivas; su convocatoria institucional de apoyo a la investigación que se realiza anualmente es muestra del interés encaminado a incentivar y estimular la inclusión de esta actividad en la labor académica no solo del docente sino de la comunidad estudiantil. En los últimos años se han efectuado estudios sobre la conceptualización de investigaciones interdisciplinarias que pueden servir de base para el conocimiento de teorías desarrolladas en este sentido; ejemplo de estas acciones es el proyecto "Pecados Capitales" vistos a través de las artes y el diseño, en el que participan arquitectos, musicólogos, diseñadores, artistas visuales y filósofos.

\section{CONCLUSIONES}

Al enfrentar de una forma muy sencilla ambos aspectos se constata que las necesidades de la investigación interdisciplinaria son acordes con las características de las artes, y específicamente del diseño, sin olvidar la gran importancia que para su desarrollo constituyen la conformación de Cuerpos Académicos, y más aun la formación de redes de trabajo, ya que representan posibilidades consistentes para la realización de este tipo de investigación; solo nos falta buscar y promover el diálogo y el trabajo en equipo entre las diversas áreas del conocimiento dentro y fuera de nuestra institución. En este sentido, el objetivo de lograr la transversalidad científica disciplinar es un reto que los investigadores de hoy debemos afrontar.

\section{REFERENCIAS BIBLIOGRÁFICAS}

Acha, J. (2006). Introducción a la teoría de los diseños. México: Trillas.

Cuerpos Académicos. Dirección de Apoyo a la Investigación y al Posgrado. Universidad de Guanajuato DAIP-DCAS. Disponible en: http://www.ugto.mx/investigacionyposgrado/promep/ca

Lizárraga, K., (2008). La falta de solidez en la formación dificulta el diálogo de disciplinas. Nexos. Boletín Informativo del Programa de Investigación Estratégica de Bolivia PIEB. 31(8), 5. Disponible en http://www. alertas-pieb.com/UserFiles/File/PDFs/NEXOS-31.pdf

Martínez, P.C., Rico Venegas, R. M. y Preciado Tarabay, S.E. (2006). Evolución de los cuerpos académicos en la Universidad de Guanajuato. Acta Universitaria. 3
(16): 15-24.

Mayorga, F. (2008). Diálogo entre disciplinas: Resultados a paso lento. En: Nexos. Boletín Informativo del PIEB, 31(8): 4-5. Disponible en: http://www.alertas-pieb. com/UserFiles/File/PDFs/NEXOS-31.pdf

Sandoval, G., (2008). Interdisciplinariedad en la investigación social. Nexos. Boletín Informativo del PIEB. 31(8):2. Recuperado de http://www.alertas-pieb.com/UserFiles/File/PDFs/NEXOS-31.pdf

Yapu, M., (2008). Investigadores e investigaciones interdisciplinarias. Nexos. Boletín Informativo del PIEB, 31(8), 3-4. Disponible en: http://www.alertas-pieb.com/ UserFiles/File/PDFs/NEXOS-31.pdf

\section{FORMA DE CITAR ESTE ARTÍCULO}

Téllez García, María Isabel de Jesús (2013). Investigación interdisciplinaria en las artes y diseño. Revista Arte y Diseño Facultad de Arquitectura, Arte y Diseño, Universidad Autónoma del Caribe, Barranquilla. ISSN 1692- 8555 Vol. 12 (N.1) PP. 30 - 36 